\title{
AVVERTENZE PER GLI AUTORI
}

Gli articoli sottoposti alla rivista vanno spediti a: Rivista Italiana di Scienza Politica, c/o Poleis - Università Bocconi, via Sarfatti 25, 20136 Milano. Essi saranno dati in lettura ai referees ed il parere della direzione sarà comunicato agli autori. Gli articoli accettati per la pubblicazione devono rispettare le convenzioni redazionali riportate di seguito.

Presentazione e formato. Gli articoli devono essere inviati su carta (in tre copie) e su dischetto. Devono essere accompagnati da un riassunto in inglese che non superi le 200 parole (anche il titolo deve essere tradotto), e da una nota bio-bibliografica di circa 60 parole, comprensiva di recapito istituzionale dell'autore e indirizzo e-mail. Gli articoli devono essere composti a spazio due, su una sola facciata con ampi margini laterali. Figure e tabelle, comprensive di note e didascalie, dovranno essere allegate e salvate in un file a parte. Nel testo deve invece risultare ben chiaro il punto dove si desidera vengano inserite. I contributi non devono superare le 12.000 parole (circa 30 pagine a stampa). Per le «Note» il limite è di 6.000 parole e per la sezione «Focus» di 3.000. In ogni caso il limite si intende comprensivo di note, tabelle, figure e riferimenti bibliografici.

Note. Il ricorso alle note a fondo pagina deve essere limitato. In generale, i riferimenti bibliografici vanno inseriti direttamente nel testo, riportando soltanto il cognome dell'autore, l'anno di pubblicazione del lavoro e - se necessario - le pagine cui ci si riferisce. Es. Easton (1953) oppure (Easton 1953) oppure (Easton 1953, 68-91). Nel caso di più di due autori, dopo il secondo si può usare l'abbreviazione et al., tutti gli altri autori vanno però citati nei riferimenti bibliografici alla fine del testo. Quando più opere dello stesso autore sono state pubblicate nello stesso anno, l'anno va contrassegnato dalle indicazioni a, b, c. Es. Rose (1974a); Rose (1974b). Nel caso di più opere dello stesso o di diversi autori, un punto e virgola separerà le diverse indicazioni. Es. (Linz 1974a; 1974b; Stepan 1978). La stessa procedura deve essere usata per le indicazioni bibliografiche contenute nelle note a fondo pagina. 
Bibliografia. Alla fine del testo i riferimenti bibliografici vanno elencati in ordine alfabetico secondo il cognome degli autori e, per ciascun autore, nell'ordine cronologico di pubblicazione delle opere ricorrendo, ove necessario, alle indicazioni a, b, c. Nel caso di lavori curati da un autore, va riportato il nome dell'autore seguito dalla dizione (a cura di), anche per i testi in lingua straniera. Il luogo di edizione di un libro va riportato nella lingua originale. Quando si riporta un'edizione originale in lingua straniera va segnalata l'eventuale traduzione italiana. I riferimenti bibliografici vanno redatti secondo regole desumibili dai seguenti esempi:

Dente, B. (a cura di) (1990), Le politiche pubbliche in Italia, Bologna, Il Mulino.

Huntington, S. (1993), The Third Wave. Democratization in the Late Twentieth Century, Norman, University of Oklahoma Press, trad. it. La terza ondata. I processi di democratizzazione alla fine del XX secolo, Bologna, Il Mulino, 1995.

Lipset, S.M. e S. Rokkan (1967), Cleavage Structures, Party Systems and Voter Alignments: an Introduction, in S.M. Lipset e S. Rokkan (a cura di), Party Systems and Voter Alignements. Cross National Perspectives, New York, The Free Press, pp. 1-64.

Inglehart, R. (1970a), Cognitive Mobilization and European Identity, in «Comparative Politics», n. 1, pp. 45-70.

- (1970b), The New Europeans: Inward or Outward Looking?, in «International Organization», n. 1, pp. 129-139

Il quadro completo e dettagliato delle convenzioni redazionali può essere trovato sul sito internet della rivista: http://www.sociol. unimi.it/risp 